\title{
Modular Countermine Payload for Small Robots
}

\section{SPIE Defense Security and Sensing}

Herman Herman

Doug Few

Roelof Versteeg

Jean-Sebastien Valois

Jeff McMahill

Michael Licitra

Edward Henciak

The INL is a

U.S. Department of Energy

National Laboratory

operated by

Battelle Energy Alliance

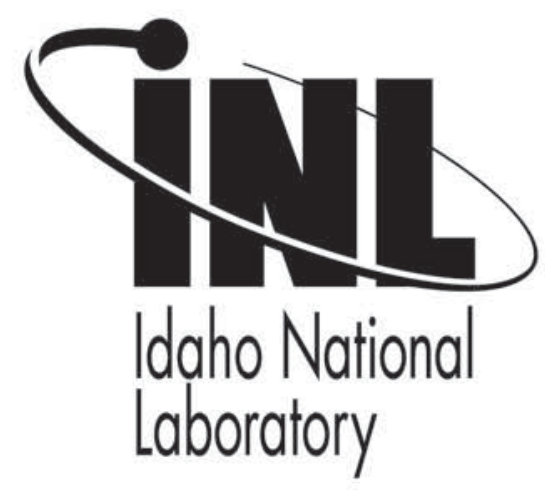

\section{April 2010}

This is a preprint of a paper intended for publication in a journal or proceedings. Since changes may be made before publication, this preprint should not be cited or reproduced without permission of the author. This document was prepared as an account of work sponsored by an agency of the United States Government. Neither the United States Government nor any agency thereof, or any of their employees, makes any warranty, expressed or implied, or assumes any legal liability or responsibility for any third party's use, or the results of such use, of any information, apparatus, product or process disclosed in this report, or represents that its use by such third party would not infringe privately owned rights. The views expressed in this paper are not necessarily those of the United States Government or the sponsoring agency. 


\title{
Modular Countermine Payload for Small Robots
}

\author{
Herman Herman ${ }^{\mathrm{a}}$, Doug Few ${ }^{\mathrm{b}}$, Roelof Versteeg ${ }^{\mathrm{b}}$, Jean-Sebastien Valois ${ }^{\mathrm{a}}$, Jeff McMahill, \\ Michael Licitra ${ }^{a}$, Edward Henciak ${ }^{\mathrm{a}}$ \\ ${ }^{a}$ Carnegie Mellon University, National Robotics Engineering Center, Pittsburgh, PA \\ ${ }^{\mathrm{b}}$ DOE INL, Idaho Falls, Idaho
}

\begin{abstract}
Payloads for small robotic platforms have historically been designed and implemented as platform and task specific solutions. A consequence of this approach is that payloads cannot be deployed on different robotic platforms without substantial re-engineering efforts. To address this issue, we developed a modular countermine payload that is designed from the ground-up to be platform agnostic. The payload consists of the multi-mission payload controller unit (PCU) coupled with the configurable mission specific threat detection, navigation and marking payloads. The multi-mission PCU has all the common electronics to control and interface to all the payloads. It also contains the embedded processor that can be used to run the navigational and control software. The PCU has a very flexible robot interface which can be configured to interface to various robot platforms. The threat detection payload consists of a two axis sweeping arm and the detector. The navigation payload consists of several perception sensors that are used for terrain mapping, obstacle detection and navigation. Finally, the marking payload consists of a dual-color paint marking system. Through the multimission PCU, all these payloads are packaged in a platform agnostic way to allow deployment on multiple robotic platforms, including Talon and Packbot.
\end{abstract}

Keywords: Payload, Countermine, robotics, human-in-the-loop, demining, teleoperated, Talon, Packbot

\section{INTRODUCTION}

Payloads for small robotic platforms have historically been designed and implemented as platform and task specific solutions. A consequence of this approach is that payloads cannot be deployed on different robotic platforms without substantial re-engineering efforts. One of the objectives of the Countermine Mobility Marking and Detection (CMMAD) effort [1] was to assess the feasibility of creating payloads which can be deployed across multiple robotic platforms and which extend the native abilities of existing robotic platforms. To this end, we developed a modular countermine payload, which can be equipped with various threat detection sensors and which can be deployed on multiple robots. This was achieved by partitioning the needed capabilities into a multi-mission payload control unit (PCU), which is platform, mission agnostic, sensor agnostic and expandable, and mission specific payloads. Together, they satisfy the CMMAD needs, but are also flexible enough to support a range of other missions. For CMMAD, the mission specific payloads consist of threat detection, marking and navigation payloads. These payloads are designed for addressing the CMMAD program's objectives as described in a companion paper [1]. While in this paper we focus on the hardware implementation of the PCU and the CMMAD payloads, we also provide a description of the associated software. This includes both the natively provided API within the PCU used for arm control, as well as the INL developed RIK/MSPC ensemble used to address the CMMAD mission.

\section{DESIGN CONSIDERATIONS AND APPROACH}

\subsection{Capability Requirements}

A typical robotic system for countermine consists of a robot, payloads (sensors, marking and navigation payloads), and a payload control system, and will have software for platform control, sensor data acquisition and data processing. While the payload control system is sometimes lumped with the payload, it fulfills a distinctly separate function. Specifically, the payload control system can be thought of as the central nervous system of the payload (and possibly also of the robot). Because of this, we separate the payload controller into a distinct module, called the multi-mission Payload Controller Unit (PCU) and into mission specific payload components. The PCU interfaces to these mission specific components to fulfill a particular mission need. To be able to do this, the PCU must have the following capabilities:

Proceedings of 2010 SPIE Defense, Security and Sensing Meeting. Conference 7664: Detection and Sensing of Mines, Explosive Objects and Obscured Targets XV. Paper 7664-47.Cite as: Herman, H, Few, D, Versteeg, R., Valois, J.V., McMahill, J., Licitra, M and Henciak, E. (2010) Modular Countermine Payload for Small Robots Proc. SPIE 7664, 7664-47 
- Flexible payload interface: since there is no one sensor that can detect all the threats, the PCU needs to be able to interface to various sensors as needed.

- Controller for sensor deployment and positioning mechanism: some threat sensors need to be swept over a suspected area, and some others need to be positioned close to the suspected target. In order to do this, the sensor payload often includes a manipulator which will need to be controlled by the PCU.

- Pose estimation: in order to perform mapping using the threat sensors and also for positioning the sensor accurately, an integrated pose sensor and estimator are needed.

- Flexible interface to robotic platforms: to accommodate various interfaces needed to control the robotic platforms, a flexible mechanical, electrical and electronic connection is needed.

In addition to the above capabilities, in order to be able to deploy the PCU across as many platforms as possible, we need to minimize the reliance of the PCU on the native robotic platform capabilities. As currently implemented, the PCU only requires two interfaces, one for power and one for control. Everything else is built into the PCU and the payloads.

While the PCU can be used to support a broad range of missions, the objective behind the creation of the PCU was to meet the CMMAD and AMDS objectives. Specifically, this required us to integrate the PCU with CMMAD mission specific payload components. These include

- Threat detection payload: this payload consists of threat sensors, such as the Cyterra AN/PSS-14 detector, and a threat sensor positioning mechanism, which in this case is a 2-axis manipulator capable of sweeping and positioning the sensor.

- Marking payload: this payload is used to mark the threat and also the area that has been cleared

- Navigation payload: this payload consists of perception sensors that are used for terrain mapping, obstacle detection and relative positioning.

The combination of the PCU and all the related payloads make up the Countermine Modular Payload for CMMAD. Due to the onboard intelligence of the PCU, the PCU and the payload can be deployed across multiple robots and with different threat sensors without the need for hardware changes.

\subsection{Operational Constraints}

Reality imposed several additional constraints on the design. The main ones are Size, Weight and Power (SWaP) constraints. As our objective is to have the robot be suitable for deployment on a small robot the size, weight and power characteristics of the payload must be minimized. These constraints were satisfied by designing a payload that can be mounted on robots as small as the Packbot while still maintaining a good battery life (about 2 hours of operational time using the current Packbot battery configuration)

A secondary constraint is on the ruggedness and environmental specification of the payload. Although the current design was only an R\&D prototype which was used in limited testing under relatively benign conditions, the objective was that it should be rugged enough to survive extended handling and testing in a range of field conditions. To satisfy this constraint, we optimized the electronics and the packaging to remove the need for active cooling, so the payload can be environmentally sealed.

\subsection{Architecture}

The requirements and constraints identified above resulted in the architecture shown in Figure 1. As discussed above, the CMMAD modular payload is self contained and accommodates multiple mission specific payloads. It includes all components needed to successfully execute a range of missions, and can accommodate multiple threat and perception sensors. Because of the self-contained nature of the payload, it is relatively straightforward to mount the payload on any robot. We only need a mechanical mount for the payload, a battery connection for power, and an interface to control the robot, which can be Ethernet, CAN or serial.

In conjunction with the modular hardware design, the software which runs on the embedded processor is also modular and adaptive. Specifically, the INL developed Real-time Intelligence Kernel (RIK) is automatically aware of which robot 
the payload is mounted on, and will adjust the representation of this robot in the OCU as well as it's behavior accordingly.

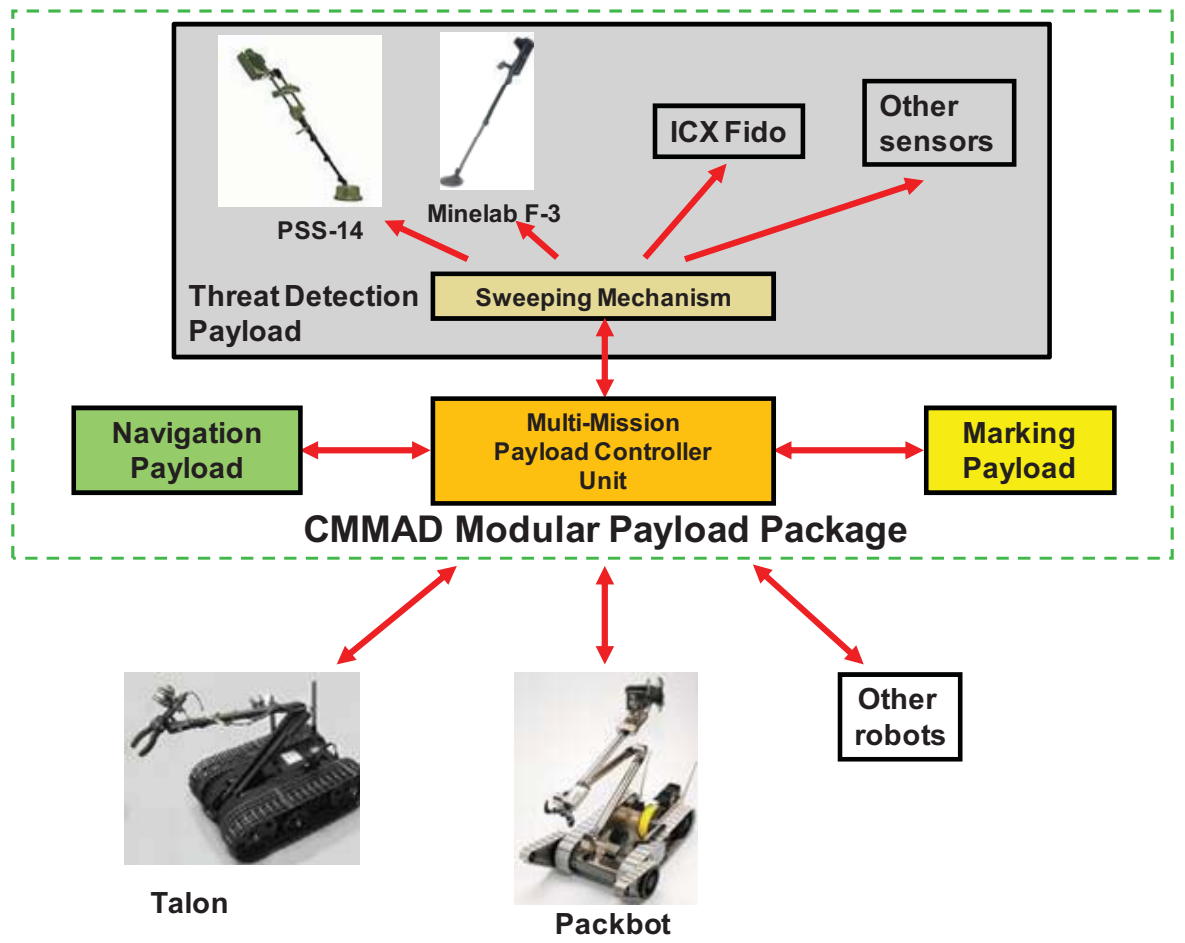

Figure 1. Block Diagram of the Payload Control Unit and the relation to other components of robotic countermine systems

\section{DETAILED COMPONENT DESCRIPTION}

As discussed above, the CMMAD payload consists of four elements which are described in more detail below:

1. The Payload Controller Unit

2. Threat detection payload

3. Marking payload

4. Navigation payload

\subsection{Payload Controller Unit}

The CMMAD Modular Countermine Payload is designed as a set of individual payloads that are controlled by the Payload Controller Unit (PCU). The PCU is also the central controller for the robot's behavior. It controls both the navigation and the mission of the robot. To achieve this objective, the PCU needs to interface to a couple mission specific payloads as depicted in Figure 1. We choose well established communication standards, such as Ethernet and USB, to connect the mission specific payloads to the PCU. By using these standards, many COTS sensors can be connected directly to the PCU. 


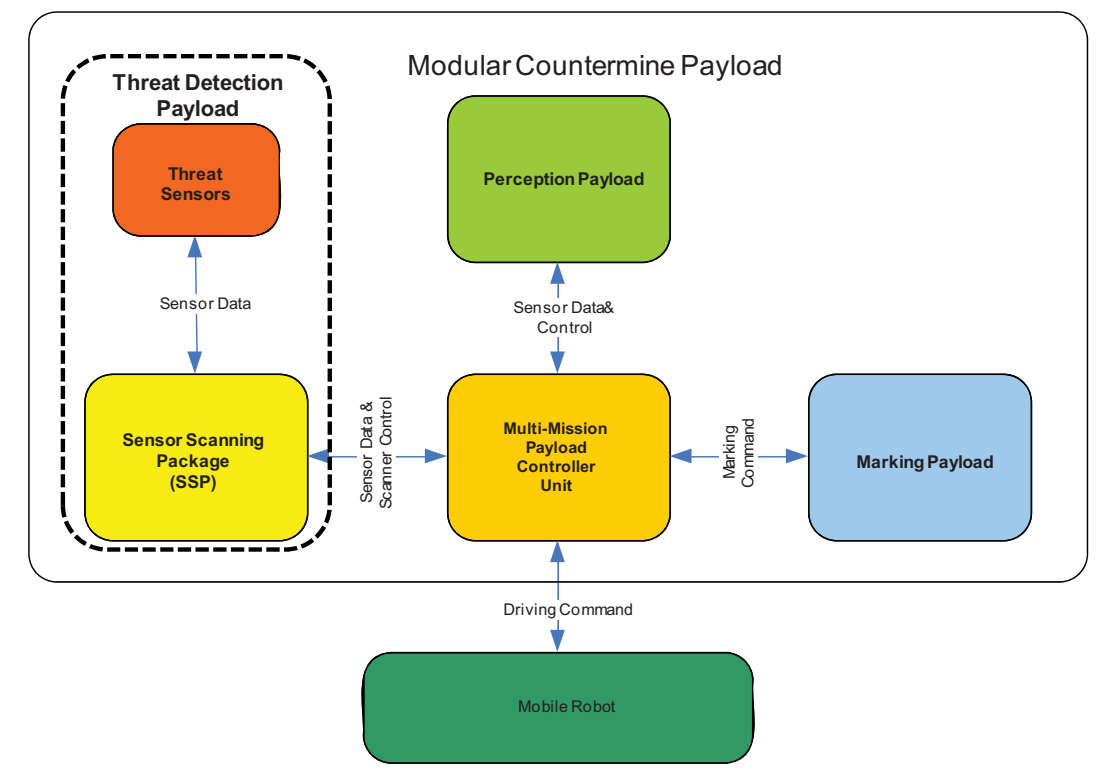

Figure 2. Functional Block Diagram of the Modular Countermine Payload

The PCU itself consists of several components:

- I/O Controller: this is a custom FPGA board with an embedded processor. It is based on the Xilinx Virtex 5 FPGA with an embedded PowerPC processor. This FPGA is chosen due to its large I/O capacity along with the inclusion of the embedded PowerPC. The richness of its $\mathrm{I} / \mathrm{O}$ enables the interfaces to the various devices, including the detector, IMU, wheel encoders and motor controllers for the sweeping mechanism, while the embedded PowerPC allows many functionalities of the $\mathrm{I} / \mathrm{O}$ controller to be programmable in $\mathrm{C}$. To further increase the modularity of the solution, we design the FPGA board as two parts. The first part is the main board, which contains the FPGA, memory, and Ethernet. The second part is the simpler I/O daughterboard that contains all the I/O transceivers, and connectors. By splitting it into the two boards, it is easier and lower in cost to add additional I/O in the future, since only the I/O daughterboard needs to be modified. The more complex main FPGA board doesn't have to be modified. In addition to interfacing to all the devices, the I/O controller also translates the I/O to Ethernet interface. Other embedded components of the I/O controller are the pose sensors, such as IMU and wheel encoders. The output of the pose sensors (e.g. wheel encoders and IMU) is combined through a pose filter to produce an estimate of the payload location and orientation.

- The E-Box embedded processor: this is the main processor for running the various software modules needed for the Modular Countermine Payload. Due to the demand on the processing power needed for running the various software modules and the constraint on power consumption, we used an ultra low voltage Core 2 Duo processor, which has an excellent power to performance ratio. The low power consumption of the processor allows it to be cooled conductively using heat-pipe, which enables the E-Box to be environmentally sealed. Despite its low power, the processor has enough processing power to execute the following software modules:

- Native software modules. These software modules were developed by CMU as part of the PCU. They include

- A software interface to the $\mathrm{I} / \mathrm{O}$ controller, which allows $3^{\text {rd }}$ party software to obtain data from the I/O controller and to command the sweeping arm through a well defined API

- A software interface to the threat detectors. This allows two way communication with the threat detector, allowing both to obtain real time data from these detectors (which can be correlated with sensor position), as well as to command the detector to perform sensor specific functions (e.g. calibration) 
- A 2D mapping module which generates a 2D map of the detector output which can be accessed by $3^{\text {rd }}$ party software

$\circ$ INL developed software. This software is essential for execution of the CMMAD mission, and includes

- Real-time Intelligence Kernel (RIK): RIK is a software module that is developed at DOE INL to address the need for semi-autonomy operation of robotic vehicles. In the payload, it serves as the high level controller which controls the behaviors of the Modular Countermine Payload. It performs multiple functions, such as terrain mapping and controlling the height of the sweep. It also interfaces to the robot and controls the navigation of the robots, so when a mine is found, it will automatically planned a new path around the mine. Finally, it also serves as the primary interface to the remote Operator Control Unit. For further reference on the behavior aspect of the project, please consult the companion paper [1] listed in the reference section.

- Mine Sensor Processing Code (MSPC): The MSPC is a standalone, modular software module which provides real time processing of threat sensor data. It provides data ingestion, map creation and real time processing. It communicates with the RIK using a high level protocol, and can be easily extended or enhanced to accommodate novel software approaches to threat detection.

- Wireless radio link, used to provide communication between the OCU and the PCU components.

- Rugged Ethernet switch used to connect the I/O controller, the E-Box, some of the sensors, and auxiliary components (including the wireless radio link)

- DC-DC converters: since various robots use different battery voltages, we need to be able to accommodate quite a wide range of power input voltages. To do this, the PCU has built-in DC-DC regulators with wide input capability (18-75V DC), allowing it to work with a wide range of voltages.

In addition to these components, there are a few additional items that are needed to deploy the payload on a robot. They include robot specific mounts and wiring harness. These items are optimized to minimize the installation time on the robot. For example, on the Talon, we built a mount that allow the sweeping mechanism to be mounted using the same mounting holes that are used to mount the original Talon manipulator. To mount the sweeping mechanism with the detector, we basically remove the original Talon manipulator and drop in the sweeping mechanism. For power we use the standard Foster Miller Y cable that can be installed in minutes. On the Packbot we use the native connections provided on the Packbot for power and Ethernet connectivity. Due to these optimizations, we can install the payload on a standard Talon or the Packbot in 30 minutes or less.

If we wanted to use a different robot for deploying this payload, the only thing required is to design a platform specific mounting adapter for the payload, built two wiring harnesses, one for supplying power to the payload and another one for communicating with the robot. We estimate that none of these platform specific items would be hard to design or expensive to build.

\subsection{Threat Detection Payload}

The threat detection payload consists of two primary components:

- Threat detection sensor: this is the sensor that is used for detecting mines or other threats. An example of this sensor is the AN/PSS-14 for mine detection and the ICX Fido for explosive detection.

- Sensor scanning mechanism: this is the manipulator that is used to sweep or position the threat detection sensor. We refer to this mechanism as the Sensor Scanning Package (SSP). It contains several subcomponents:

- Sweeping mechanism: a 2-axis manipulator which is designed specifically for sweeping and positioning the threat detection sensor. In the future, this manipulator can be replaced with a 4-6 
degrees manipulator that would allow more flexibility in term of sweeping and positioning the sensor in constrained spaces. One very useful feature in the design is the central hollow mast in the middle of the sweeping mechanism. The hollow mast allows wiring and paint plumbing to be routed through the center of the mast. In addition, since the center mast does not rotate with the sweeping mechanism, it also serves as a nice mounting location for the video and ladar sensors that we use for remote monitoring and terrain mapping.

○ Motor controllers: these are the controllers for the sweeping mechanism. They control the fail-safe brake that will activate when the E-Stop button is pressed, or if there is a power failure. The motor controllers have built-in current limiters which are used as a pseudo force sensors. This is very useful for detecting collision of the detector with objects in the environment, and is also used for tapping the ground to determine the ground height. The motor controllers are designed for precise closed-loop control of the sweeping mechanism, to maximize the sensor detection performance.

○ Sensor mounting adapter: a mechanical adapter to mount the sensor on the sweeping mechanism

\subsection{Marking Payload}

The marking payload is a dual color paint marking system that is designed and built by SPAWAR. It has two paint tanks that can be filled with two different colors, one for marking the lane boundary for the path that the robot has swept, and the other one for marking the threat, such as mines. The paints are routed through three separate nozzles. The two nozzles for the lane marking are mounted on two short arms. The distance between the two nozzles is identical to the sweeping width of the detector. The threat marking nozzle is mounted near the detector's head. The payload is designed so it can be interfaced and controlled over Ethernet, which simplifies interconnectivity.

\subsection{Navigation Payload}

The navigation payload supplies the perception capability that is needed to navigate autonomously. It consists of several perception and global pose sensors which are interfaced through the PCU interfaces:

- Sick LMS100 scanning ladar: this ladar unit is used for relative positioning and also for obstacle detection and avoidance

- Hokuyo UTW-30LX scanning ladar: this ladar unit is used for terrain mapping. The resulting terrain map is utilized for controlling the sweep height of the threat detection sensor.

- Video camera: this is an optional component that is used to give the robot operator remote video feed for situational awareness.

- GPS and IMU: this is an optional component that can measure the GPS coordinate of the robot and the sensor. It is equipped with a high-end IMU to allow operation in GPS denied area.

\section{IMPLEMENTATION}

The design and actual construction of the PCU and associated payload components occurred as part of the CMMAD program. Three complete systems were constructed, which can be deployed on either the Talon or Packbot. Apart from the two different mounts for the Talon and Packbot these systems are identical. As part of the implementation effort the overall mounting schemes was optimized and field tested. On either robot, we can mount the payload on each robot in less than half an hour. Figure shows the PCU and payload components mounted on the Talon and the Packbot. 

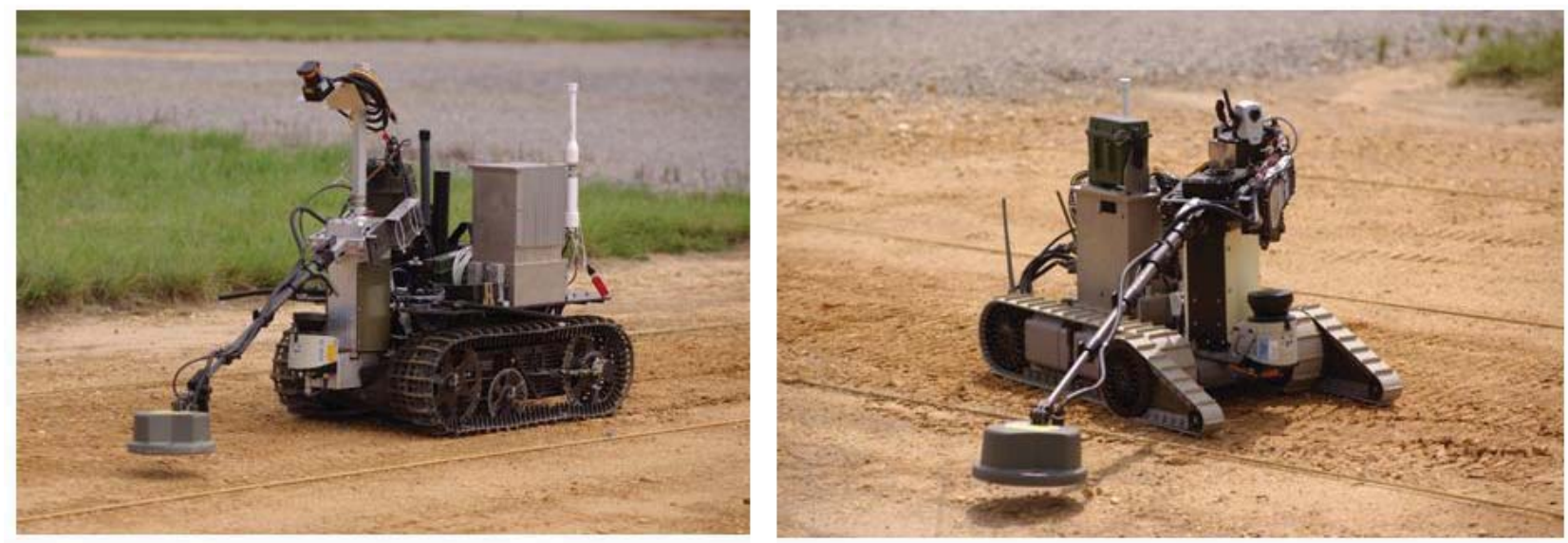

Figure 3. The Modular Countermine Payload mounted on the Talon and the Packbot. Other than the difference in the mounts, both payloads are identical.

Figure 4 shows the payload mounted on the Talon robot with annotations on its various components. The figure shows the configuration of the payload with the AN/PSS-14 detector. Figure 5 shows the payload configured with the ICX Fido explosive sensor. It takes about 15 minutes to swap the AN/PSS-14 with the ICX Fido, so the swap is fast enough to be done in the field. The sweeping mechanism is also fast enough for optimally sweeping the AN/PSS-14 and precise enough for positioning the ICX Fido sensor.

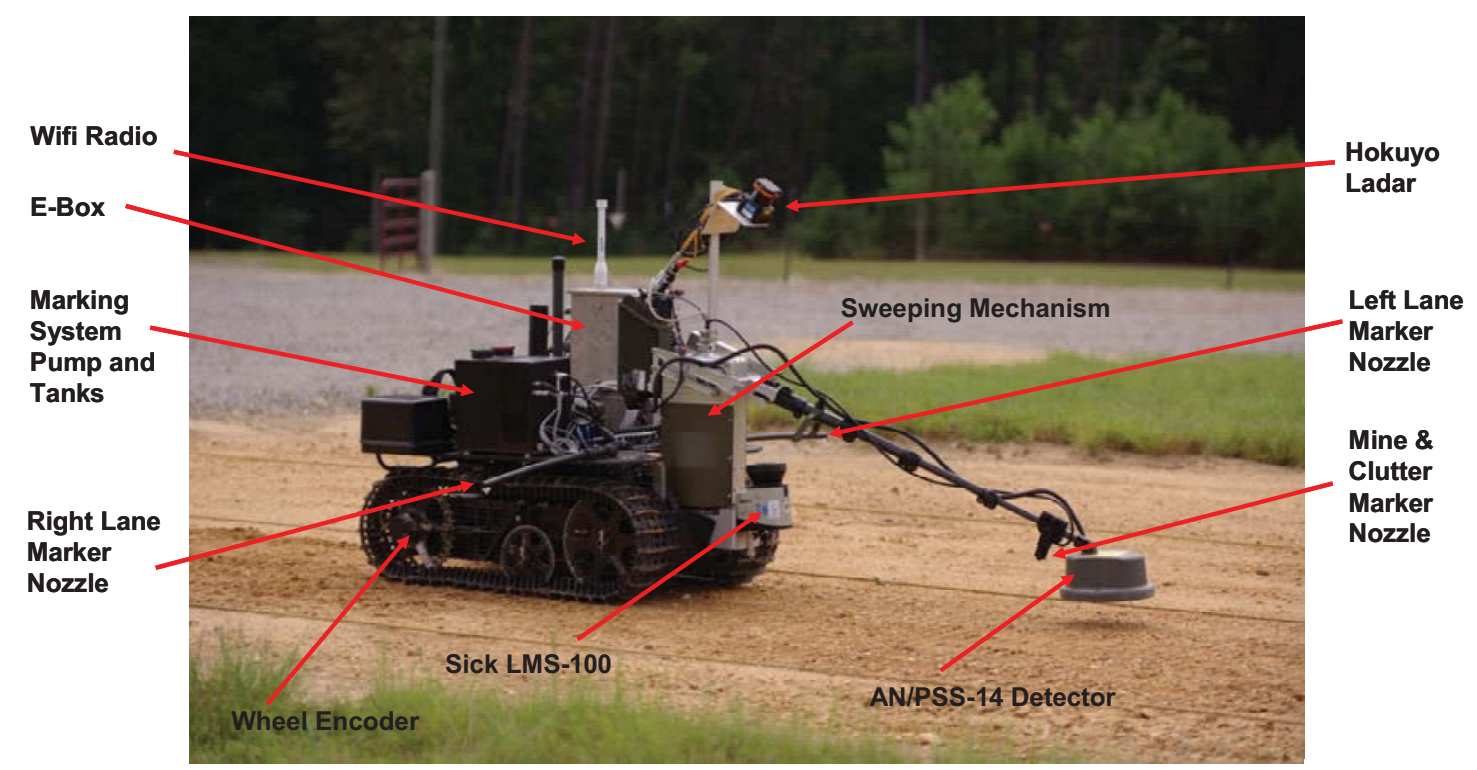

Figure 4. The Talon robot with the Modular Countermine Payload on a mine lane 


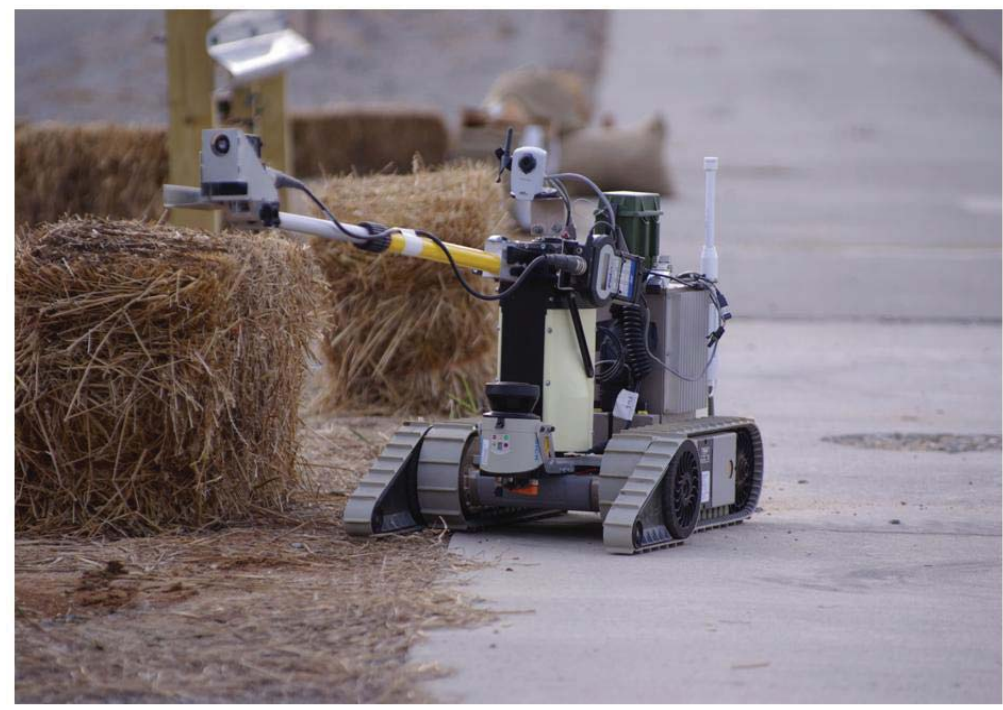

Figure 5. The payload with the ICX Fido sensor mounted on the Packbot

As briefly mentioned before, one key factor which enables the plug and play nature of the payload is the capability of the software (both the native, CMU provided and the INL provided RIK/MSPC combination) to adapt to the various detectors and robots. For example, the low level software on the SSP FPGA board can utilize the external wheel encoders, or it can accept speed measurement from the robot, and this choice happens automatically. Another example is the RIKs ability to automatically detect which robot it is operating on, and make the necessary internal configuration changes to enable correct operation on that platform.

Another key factor is the fact that the PCU does not rely on anymore than the most basic capabilities of the host robot. Specifically, the host robot doesn't need to have any pose measurement capability, but rather, the PCU provides pose data in association with threat data. This can then be used for map generation (Figure 6). This data shows the locations and shape of the threat sensor data.

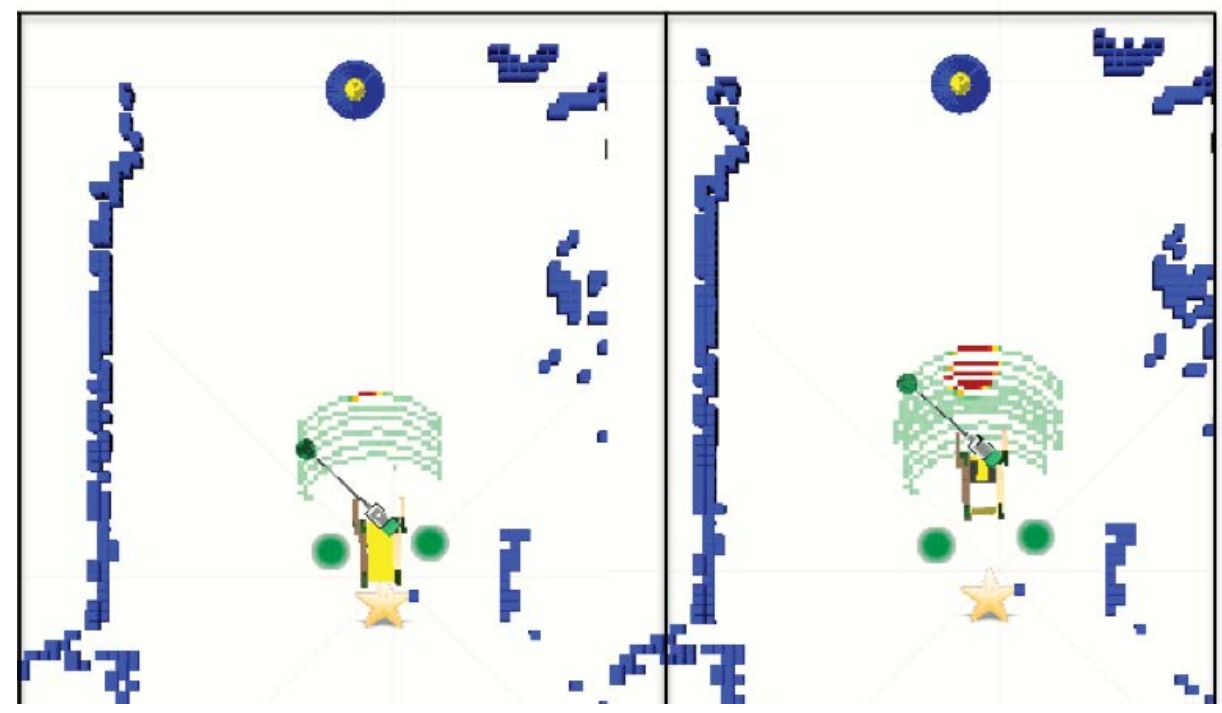

Figure 6. Sample output from the CMMAD Payload. The blue dots signify obstacles, the green dots signify sweeping path, and the red dots signify a strong EMI response (from [1]). The PCU provides the pose and sensor data without having to rely on the robot to provide pose information. 


\section{CONCLUSION AND FUTURE DIRECTION}

By formally separating the payload into a Payload Control Unit and mission specific payload components we developed a flexible system which can be deployed across multiple robots with multiple sensors. We used this approach to rapidly develop several field validated instances of modular payload for countermine tasks which were deployed on the Packbot and Talon. Our approach segmented functionality in different modular hardware and software components with well defined responsibilities and interfaces.

The platform and sensors agnostic nature of the payload implies the applicability of this approach (and the associated components) to future generations of platforms and sensors. Modifications are likely to be needed to several of the components to accommodate future program needs, such as the need to accommodate array based detectors which need more degrees of freedom to be manipulated in an effective manner, and the need to accommodate novel processing algorithms. However, as such modifications only would affect discrete modules in our system (and not the overall structure), we are confident that our design is well suited to support future AMDS needs.

There are also a couple general improvements that will further enhance the payload. The first one is an improvement in form factor. Although the current payload is small enough to be mounted on the Talon and Packbot, it can still be optimized. We believe we can reduce the size and weight by about $30 \%$ if we make another design iteration. The second improvement would on the design of the sweeping mechanism to make it more dexterous.

\section{ACKNOWLEDGEMENTS}

Funding for the CMMAD effort was provided through the AMDS program which was administered through US Army RDECOM CERDEC Night Vision and Electronic Sensors Directorate. We acknowledge the support and guidance of the program manager (Ed McAuley) as well as that of the Night Vision team (Mark Locke, Eloisa Lara and Matt Aiello) and the IDA team. We also acknowledge the support and help from SPAWAR which designed and built the paint marking module.

\section{REFERENCES}

[1] 1. D. Few, R. Versteeg and Herman, H.. Semiautonomous Mine Detection System. in 2010 SPIE Defense Security and Sensing Meeting. 2010. Orlando, Florida.

[2] D. Anderson, T. Howard, D. Apfelbaum, H. Herman. Coordinated Control and Range Imaging for Mobile Manipulation. Proceedings of the 11th International Symposium on Experimental Robotics 2008 (ISER '08), August, 2008

[3] H. Herman, J. McMahill. Portable robotic platform for handheld landmine detection system. in 2004 SPIE Defense Security and Sensing Meeting. 2004. Orlando, Florida 